\title{
Anesthetic care for electroconvulsive therapy during pregnancy -A case report-
}

\author{
Hong-Seuk Yang, Hye-Jeong Seo, and Yoon Kyung Lee
}

Department of Anesthesiology and Pain Medicine, Asan Medical Center, College of Medicine, University of Ulsan, Seoul, Korea

Electroconvulsive therapy (ECT) is recommended for patients with psychotic disorders that do not respond to medication. Usually, many psychotropic medications have teratogenic effects on the fetus. ECT seems to be a relatively safe and effective treatment during pregnancy. We report here on a 33-year-old primigravida patient in her third trimester receiving ECT. (Korean J Anesthesiol 2011; 60: 217-220)

Key Words: Anesthetic care, Electroconvulsive therapy, Pregnancy.

Electroconvulsive therapy (ECT) is recommended for patients with bipolar disorder who do not respond to medications [1]. It is safe and its efficacy has been well-established. Moreover, many psychotropic medications are known to have teratogenic effects on the fetus, and therefore ECT seems to be a relatively safe and effective choice of treatment in pregnant patients [2]. We experienced a pregnant patient in her third trimester receiving ECT and describe the results.

\section{Case Report}

A 33-year-old primigravida at 28 weeks of gestation was admitted to a Psychiatric Department because of irritability, insomnia, and poor oral intake. She was not as talkative as she used to be and became paranoid. These symptoms started approximately 2 weeks prior to admission. The patient was diagnosed with schizophrenia 15 years ago. Since then she has been hospitalized five times with psychotic symptoms. The patient had been taking $3 \mathrm{mg}$ of risperidone, $2 \mathrm{mg}$ of benztropine, $10 \mathrm{mg}$ of zolpidem, $25 \mathrm{mg}$ of trazodone, and $25 \mathrm{mg}$ of quetiapine everyday prior to admission.

She was diagnosed as having a bipolar disorder with depressive episode and olanzapine $5 \mathrm{mg}$ /day was administered additionally. Psychiatric examination revealed lack of self-care, and decreased psychomotor activity. Physical and neurological examinations were within normal limit.

Electroconvulsive therapy was recommended and informed consent was obtained from her and her family. The obstetrician diagnosed her as $28^{+1}$ weeks of pregnancy. The fetus was small for gestational age ( $0.77 \mathrm{~kg}$ estimated fetal body weight),

Received: September 1, 2010. Revised: October 7, 2010. Accepted: October 8, 2010.

Corresponding author: Hong-Seuk Yang, M.D., Department of Anesthesiology and Pain Medicine, Asan Medical Center, College of Medicine, University of Ulsan, 388-1, Pungnap-dong, Songpa-gu, Seoul 138-736, Korea. Tel: 82-2-3010-3865, Fax: 82-2-470-1363, E-mail: hsyang@amc.seoul.kr (c) This is an open-access article distributed under the terms of the Creative Commons Attribution Non-Commercial License (http:// creativecommons.org/licenses/by-nc/3.0/), which permits unrestricted non-commercial use, distribution, and reproduction in any medium, provided the original work is properly cited. 
but the location of placenta, amount of amniotic fluid and fetal heart rate were reported within the normal range. No other abnormality in her obstetric ultrasound was observed. Laboratory tests including $\mathrm{CBC}$, and chemical test and electrocephalogram were within normal limit, except for hypoalbuminemia with an albumin level of $2.5 \mathrm{~g} / \mathrm{dl}$. There was no sign of premature labor and the pregnancy was uneventful.

The patient was scheduled to undergo ECT seven times during 2 weeks. The electrocardiography, pulse oximetry and noninvasive blood pressure were measured during every ECT application. The fetal heart rate and uterine contractility were monitored by ultrasound during ECT and recovery periods. The patient also had a pad under the right side hip for left uterine displacement.

Initial vital signs were stable, blood pressure was $100-$ 120/60-80 $\mathrm{mmHg}$, heart rate was $80-90 \mathrm{bpm}$ and oxygen saturation was $97-100 \%$ on room air.

Anesthesia was induced with thiopental ( $4 \mathrm{mg} / \mathrm{kg}$ ) without inhalation of any volatile anesthetics and muscle relaxation was obtained by succinylcholine $(1 \mathrm{mg} / \mathrm{kg}$ ) with Sellick's maneuver. The patient was ventilated manually with $100 \%$ oxygen $3 \mathrm{~L} /$ min using a face mask. Electrical stimulation was provided with a charge of $168 \mathrm{mC}$ and the seizure duration was 75 second. Fetal heart rate was $140-160 \mathrm{bpm}$, and regarded as normal variability during ECT. After ECT, the patient stayed in recovery room for an hour to monitor both the mother and fetus. One hour after the first session of ECT, we noticed an abnormal movement in the patient's abdomen. Fetal ultrasound was performed and regular uterine contraction was found. Fetal heart rate was $120-160 \mathrm{bpm}$. It was regarded as preterm labor, so we administered $50 \mathrm{mg}$ of ritodrine mixed with $5 \%$ dextrose $500 \mathrm{ml}$. Two hours later, the uterine contraction disappeared in the recovery room. After the patient's state improved to normal, she was moved to the general ward.

The patient underwent another six sessions of ECT. The electrical stimulations in the second, third and fourth sessions were $172 \mathrm{mC}$ and seizure durations were $45 \mathrm{sec}, 27 \mathrm{sec}$, and $20 \mathrm{sec}$. The electrical stimulation in fifth, sixth and seventh sessions was $288 \mathrm{mC}$, and seizure durations were $45 \mathrm{sec}, 30$ sec, and $34 \mathrm{sec}$. After the sixth session, psychiatric symptoms were improved markedly, and two weeks later, the patient was discharged with $7.5 \mathrm{mg}$ /day of olanzapine.

Three weeks after discharge, the patient was readmitted with the recurred psychiatric symptoms, and emergency cesarean section was performed. She was delivered of a $35^{+4}$ weeks old premature baby weighing $1,940 \mathrm{~g}$. The baby was diagnosed with hyaline membrane disease and congenital hypertrophic pyloric stenosis. He received ventilatory care for 7 days with surfactant treatment, and two month later he was operated on with the pyloromyotomy procedure.

\section{Discussion}

About $7 \%$ of women of childbearing age suffer from acute symptoms of moderate to severe mental illness, or have recently experienced an episode requiring drug treatment [3]. Psychotropic medications are the first choice in treatment of mood disorder with psychotic features occurring in pregnant women [4]. But the psychotropics most commonly used for treating bipolar disorder are known to have adverse effects when used during pregnancy and breastfeeding periods [5].

ECT is a nonpharmacologic biological treatment which has been shown to be a highly effective treatment option, especially for depression, and also for psychotic manifestation of schizophrenia [1]. In view of its efficacy and speed of action, ECT may be a first-line treatment for life-threatening depression and a second line treatment for patients with major depressive disorders who do not respond or respond incompletely to antidepressant drugs [1].

ECT is now performed under general anesthesia. Guidelines by the American Psychiatric Association stated that anesthesia for ECT should be administered by a specially trained anesthesiologist, and the anesthesiologists should have overall responsibility, not only for anesthesia itself, but also for cardiopulmonary management and emergency care [6].

To optimize the anesthetic management of patients undergoing ECT, it is important to understand the physiologic responses to the electrical stimulus, the effect of anesthetic drugs on the ECT response, and the pharmacologic effects of the drugs used to attenuate side effects related to ECT [7].

Hemodynamic responses that occur during the ECT consist of generalized autonomic nervous system stimulation [7]. As the electrical current is delivered, the parasympathetic nervous system stimulation results in bradycardia, or rarely, asystole. Shortly thereafter, the sympathetic nervous system is stimulated, which usually causes tachycardia, hypertension lasting 5 minutes or longer, and may lead to arrhythmias $[7,8]$. Increased catecholamine blood level also can affect the uterus [9]. Vasoconstriction from catecholamine action decreases blood flow to the uterus and placenta with potential hypoxemia of the fetus. Usually, transient hypoxemia is tolerable. However, fetal bradycardia can occur if there are preexisting conditions such as chronic hypertension, or diabetes which tend to compromise uteroplacental circulation [9]. In this patient, transient hypertension $(160-180 / 100-120 \mathrm{mmHg})$ and tachycardia (130-140 bpm) occurred during seizure activity, but fetal heart rate was maintained (140-160 bpm) during that period.

The efficacy of ECT in alleviating acute depression is dependent on the duration of the induced seizure. EEG seizure activity lasting from 25 to 50 seconds is alleged to produce 
the optimal antidepressant response. Patients experiencing seizure duration of shorter than 15 seconds or longer than 120 seconds achieve a less favorable response to ECT. Since many of the anesthetic drugs used for ECT have anticonvulsant properties, they would be expected to decrease the duration of ECT-induced seizure activity in a dose-dependent manner [7]. Methohexital is considered the "gold standard" in anesthetics for ECT $[6,7]$. It does not affect ECT seizure duration. Thiopental sodium can be an alternative barbiturate although it decreases seizure duration. Propofol has been used in many reports, because systemic cerebrovascular and hemodynamic changes under propofol anesthesia are more stable than under barbiturate anesthesia $[8,10]$. Volatile anesthetics such as sevoflurane can also be used in ECT. However, its timeconsuming induction, depression of seizure duration, and the requirement of an anesthesia machine make this technique impractical, except for women requiring ECT in the late stages of pregnancy, when it may reduce post-ECT uterine contractions $[7,10]$. Succinylcholine is the most commonly used muscle relaxant in ECT because of its short action duration. In this case, anesthesia was induced with thiopental sodium and succinylcholine. EEG seizure durations lasted for 20-75 seconds, and the psychotic symptoms improved.

Benabarre et al. [11] suggests that ECT minimize the risk for both mother and fetus. However, studies on the use of ECT during pregnancy, which is effective and appears to be safe, are limited [12]. Anderson and Reti [12] reviewed 339 cases on the use of electroconvulsive therapy during pregnancy from 1941 to 2007. It seems that ECT is an effective treatment for severe mental illness during pregnancy and that the risks to fetus and mother are low. But there were 20 maternal complications reported and 18 were likely related to ECT such as uterine contraction and/or preterm labor, vaginal bleeding, miscarriage, status epilepticus, hematuria, abdominal pain, and placental abruption. Fetal adverse events also reported 25 cases, which included fetal death, transient fetal bradycardia and/or decelerations, club foot, prematurity, congenital blindness, aorta coarctation, cortical infarcts, VATER syndrome and mental retardation. Of the 11 reported fetal deaths, only one was believed to be the direct result of ECT, which followed an episode of status epilepticus in the mother secondary to ECT [12]. We suspect that hyaline membrane disease and congenital hypertropic pyloric stenosis shown in this case were not related with ECT, but were not able to completely exclude in this possibility.

Another survey of 318 pregnant women observed nine cases of premature labor caused by ECT [11]. We also found that uterine contraction appeared about 1 hour after the first session, and was controlled by ritodrine. In the next 6 sessions of ECT there was no episode of uterine contraction or other adverse events. Close monitoring of maternal and fetal conditions after cessation of ECT could have helped to detect adverse events probably caused by ECT in advance.

Uterine contraction usually can be controlled by ritodrine or magnesium sulfate as in this case, and fetal bradyarrhythmias can be avoided by adequate preoxygenation and proper position of the mother to minimize aortocaval compression by elevating the right hip and displacing the uterus $[12,13]$.

Another anesthetic consideration in ECT for pregnancy is on regurgitation of gastric acid resulting from an upward displacement of the stomach and intestines by the enlarged uterus [9]. The simplest method to prevent aspiration is to apply constant manual pressure to the cricoid cartilage (Sellick's maneuver). Patients in their third trimester should be considered for endotracheal intubation with ECT. We had the patient keep the NPO for more than 8 hours with fluid infusion and Sellick's maneuver was applied during ECT.

Although ECT typically has little effect on fetal and maternal status [14], the anesthesiologist also should be aware of the complications of the ECT. Tocodynamometry during ECT can be helpful for monitoring uterine activity and early detection of uterine contraction [15].

This patient received a Cesarean-section due to aggravating psychotic symptoms. The baby was born prematurely with under-development of the pulmonary system and congenital hypertropic pyloric stenosis. This baby got the ventilatory care with surfactant treatment and pyloromyotomy.

On first ECT application, uterine contraction was detected after 1 hour of ECT, which was well controlled with a tocolytic agent. In anesthetic care for ECT during pregnancy, monitoring of the mother and fetus for over 1 hour is important with airway management during ECT.

\section{References}

1. Baghai TC, Möller HJ. Electroconvulsive therapy and its different indications. Dialogues Clin Neurosci 2008; 10: 105-17.

2. Miller LJ. Use of electroconvulsive therapy during pregnancy. Hosp Community Psychiatry 1994; 45: 444-50.

3. Bozkurt A, Karlidere T, Isintas M, Ozmenler NK, Ozsahin A, Yanarates $\mathrm{O}$. Acute and maintenance electroconvulsive therapy for treatment of psychotic depression in a pregnant patient. J ECT 2007; 23: 185-7.

4. Altshuler LL, Cohen L, Szuba MP, Burt VK, Gitlin M, Mintz J. Pharmacologic management of psychiatric illness during pregnancy: dilemmas and guidelines. Am J Psychiatry 1996; 153: 592-606.

5. Dodd S, Berk M. The pharmacology of bipolar disorder during pregnancy and breastfeeding. Expert Opin Drug Saf 2004; 3: 221-9.

6. American Psychiatric Association Committee on Electroconvulsive Therapy. The practice of electroconvulsive therapy: recommendations for treatment, training, and privileging. 2nd ed. Washington, DC, American Psychiatric Association. 2001.

7. Ding Z, White PF. Anesthesia for electroconvulsive therapy. Anesth 
Analg 2002; 94: 1351-64.

8. Mayo C, Kaye AD, Conrad E, Baluch A, Frost E. Update on anesthesia considerations for electroconvulsive therapy. Middle East J Anesthesiol 2010; 20: 493-8.

9. Walker R, Swartz CM. Electroconvulsive therapy during high-risk pregnancy. Gen Hosp Psychiatry 1994; 16: 348-53.

10. Saito S. Anesthesia management for electroconvulsive therapy: hemodynamic and respiratory management. J Anesth 2005; 19: 142-9.

11. Benabarre A, Bernardo M, Arrufat F, Salvà J. Management and treatment of severe mental disorders in pregnancy. Actas Esp Psiquiatr 2000; 28: 45-58.

12. Anderson EL, Reti IM. ECT in pregnancy: a review of the literature from 1941 to 2007. Psychosom Med 2009; 71: 235-42.

13. Impastato DJ, Gabriel AR, Lardaro HH. Electric and insulin shock therapy during pregnancy. Dis Nerv Syst 1964; 25: 542-6.

14. Repke JT, Berger NG. Electroconvulsive therapy in pregnancy. Obstet Gynecol 1984; 63: 39S-41S.

15. Kasar M, Saatcioglu O, Kutlar T. Electroconvulsive therapy use in pregnancy. J ECT 2007; 23: 183-4. 